\title{
Joint Channel and Carrier Estimation Using Extended Kalman Filter
}

\author{
Grzegorz Haza
}

\begin{abstract}
In this paper a proposal of joint channel and carrier estimation using extended Kalman filter (EKF) is presented. For the proposed algorithm simulations are performed and the results are compared with the separate channel estimation based on the Kalman filter (KF) and carrier estimation based on the extended Kalman filter (EKF).
\end{abstract}

Keywords-extended Kalman filter, EKF, joint channel and carrier estimation, synchronization, digital receiver.

\section{INTRODUCTION}

$\mathbf{E}$ STIMATION and synchronization of communication signal parameters is an important task in a design of coherent receivers that consume significant attention of engineers. A common approach for estimation of an impulse response of a communication channel and a residual carrier frequency offset is based on a disjoint adaptive Least Mean Squares (LMS) channel estimation [1]-[4] with frequency synchronization based on a phase locked loop (PLL) [1], [2], [5], [6] with an appropriate phase error detector (PED) [1], [2], [5], [6].

However, this technique suffers from the fact, that both algorithms are based on a fixed adaptation parameters which, in case of the LMS channel estimation is represented by an adaptation step [1], [3], [4] and for the PLL carrier synchronization, by a loop filter parameters related with the loop bandwidth [1], [2], [5], [6]. As a result of fixed step-size adaptation procedure, the algorithms must be preconfigured in order to provide quick adaptation or small value of estimation error in the steady state. These both contradictions can not be met for its best performance at the same time. Since the adaptation steps do not depend on the actual conditions of the received signal, it is often a compromise between a maximum acceptable time of adaptation and a desired steady state error estimation.

Because of this, more advanced adaptive techniques for the channel estimation, for instance based on the RLS algorithm [1], [3], [4] or the Kalman filter [1], [3], [4] and for the phase estimation and synchronization, techniques based on a variable step PLL [7], on the Kalman filter with an appropriate phase detector [8] or on the extended Kalman filter [9] were proposed.

In this paper, a joint channel and carrier estimation based on the extended Kalman filter (EKF) is proposed. This technique relies on the concept of applying the EKF for a carrier frequency estimation presented in [9]. However, we use a statespace nonlinear dynamical model that incorporates both, the carrier frequency and the finite impulse response (FIR) type

G. Haza is with the Institute of Telecommunications Teleinformatics and Electronics, Wrocław University of Technology, Wybrzeże Wyspiańskiego 27, 50-370 Wrocław (e-mail: grzegorz.haza@pwr.wroc.pl). channel parameters at once in an iterative estimation procedure.

The paper is organized as follows. At the beginning, the state-space model description for complex signals and then a representation with separated real and imaginary parts is provided. Next, the application of the Extended Kalman Filter for described state-space model is proposed and later on, simulation results are shown and described. Finally, some conclusions are given at the end of this paper.

\section{MODEL DESCRIPTION}

\section{A. State-space Model a Nonlinear Dynamical System}

Assume, that the input signal $y(t)$ at the receiver is at first downconverted to the baseband representation with the residual carrier frequency offset (CFO), then matched filtered and sampled with perfect timing information with time step $T_{s}=1 / f_{s}$ for one sample per symbol, so the following discrete time complex representation can be applied [1], [5]:

$$
y_{n}=\sum_{k=0}^{L-1} h_{k} b_{n-k} \exp \left\{j \omega_{0} n T_{s}\right\}+v_{n}
$$

where $\mathbf{h}=\left[h_{0}, \ldots, h_{k}, \ldots, h_{L-1}\right]^{T}$ is the finite impulse response channel type of length $L$ introducing inter-symbol interference, which is assumed to be complex valued so, that $h_{k} \in \mathcal{Z}$ for $k=0, \ldots, L-1$. Transmitted symbol $b_{n} \in \mathcal{Z}$ is assumed to be independent and identically distributed random variable (iid RV) with uniformly distributed symbols taken from $m$-PSK or $m$-QAM constellation [1], [2], [5], [6]. The exponential component $\exp \left\{j \omega_{0} n T_{s}\right\}$ is responsible for the multiplicative distortion [1] with a frequency offset value $\omega_{0} \in \mathcal{R}$ and time-variable phase $\phi_{n}=\omega_{0} n T_{s} \in \mathcal{R}$, which can be caused i.e., by an imperfect carrier frequency of generators at the sending and receiving end, a Doppler frequency offset or both [1], [2], [5], [6]. The additive noise term $v_{n}$ is assumed to be complex valued, zero-mean, white Gaussian noise (Additive White Gaussian Noise - AWGN).

Our goal is to perform an iterative and joint estimation of the carrier phase $\phi_{n} \in \mathcal{R}$, the carrier frequency offset $\omega_{n} \in \mathcal{R}$ and the channel impulse response $\mathbf{h}_{n}$ of length $L$ where $\mathbf{h}_{n}[k] \in \mathcal{Z}$ for $k=0, \ldots, L-1$, using the extended Kalman filter, which operates in the state-space model of a nonlinear dynamical system.

In order to achieve this goal, we assume at first a process equation of the dynamical system described as below

$$
\mathbf{x}_{n}=\mathbf{A} \mathbf{x}_{n-1}+\mathbf{w}_{n}
$$

where the process equation at time instant $n$ and $n-1$ contains consecutively $\mathbf{x}_{n}=\left[\begin{array}{lll}\phi_{n}, & \omega_{n}, & \mathbf{h}_{n}^{T}\end{array}\right]^{T}$ and 
$\mathbf{x}_{n-1}=\left[\phi_{n-1}, \omega_{n-1}, \mathbf{h}_{n-1}^{T}\right]^{T}$. A transition matrix of the process equation is of size $L+2 \times L+2$ and it is described as follows

$$
\mathbf{A}=\left[\begin{array}{ccc}
1 & T_{s} & \mathbf{0}_{1 \times L} \\
0 & 1 & \mathbf{0}_{1 \times L} \\
\mathbf{0}_{L \times 1} & \mathbf{0}_{L \times 1} & \mathbf{A}_{\mathbf{h}}
\end{array}\right]
$$

where the channel propagation matrix is defined as $\mathbf{A}_{\mathbf{h}}=\mathbf{I}_{L \times L}$. A process noise $\mathbf{w}_{n}=\left[w_{\phi, n}, w_{\omega, n}, \mathbf{w}_{\mathbf{h}, n}^{T}\right]^{2}$ is a vector of size $L \times 1$, where $w_{\phi, n} \in \mathcal{R}$ and $w_{\omega, n} \in \mathcal{R}$ but, on the other hand, an additive channel model noise is assumed to be $\mathbf{w}_{\mathbf{h}, n}[k] \in \mathcal{Z}$ for $k=0, \ldots, L-1$. The process noise $\mathbf{w}_{n}$ is assumed to be additive, zero mean, white and Gaussian [4], [10]. With the aforementioned representation, both the carrier frequency offset and the channel characteristic are included in the vector representation of the process equation of the dynamical system.

For the EKF purposes, assuming the AWGN measurement noise $v_{n}$, a general measurement equation of the nonlinear state-space dynamical model, which is related to the process equation $\mathbf{x}_{n}$ and the vector $\mathbf{b}_{n}=\left[b_{n}, b_{n-1}, \ldots, b_{n-L+1}\right]^{T}$ of size $L \times 1$ with transmitted symbols, can be described by the following formula [10]

$$
y_{n}=c\left[\mathbf{x}_{n}, \mathbf{b}_{n}\right]+v_{n}
$$

Between the process and measurement equations a nonlinearity represented by the multiplicative distortion [1], that includes the phase $\phi_{n}$ of the process equation $\mathbf{x}_{n}$ is present. This nonlinearity can be placed into the aforementioned formula by the following complex relation

$$
c\left[\mathbf{x}_{n}, \mathbf{b}_{n}\right]=\mathbf{h}_{n}^{T} \mathbf{b}_{n} \exp \left\{j \phi_{n}\right\}
$$

In order to enable state estimation for the nonlinear measurement equation using the Extended Kalman Filter, it is necessary to perform linearisation by expanding the nonlinearity into the first order Taylor series [4], [10] at the reference point $\mathbf{x}_{n, \text { ref }}$ according to the following approximation

$$
c\left[\mathbf{x}_{n}, \mathbf{b}_{n}\right] \approx c\left[\mathbf{x}_{n, r e f}, \mathbf{b}_{n}\right]+\frac{d c\left[\mathbf{x}_{n, r e f}, \mathbf{b}_{n}\right]}{d \mathbf{x}_{n, r e f}^{T}} \delta \mathbf{x}_{n}
$$

where $\delta \mathbf{x}_{n, r e f}$ is the state perturbation, which can be represented by

$$
\delta \mathbf{x}_{n, r e f}=\mathbf{x}_{n}-\mathbf{x}_{n, r e f}
$$

and the first derivative in (6) is the Jacobian of size $1 \times L+2$, which can be restated as

$$
\begin{aligned}
\mathbf{C}\left[\mathbf{x}_{n, r e f}, \mathbf{b}_{n}\right] & =\left.\mathbf{C}\left[\mathbf{x}_{n}, \mathbf{b}_{n}\right]\right|_{\mathbf{x}_{n}=\mathbf{x}_{n, r e f}} \\
& =\left.\frac{d c\left[\mathbf{x}_{n}, \mathbf{b}_{n}\right]}{d \mathbf{x}_{n}^{T}}\right|_{\mathbf{x}_{n}=\mathbf{x}_{n, r e f}}
\end{aligned}
$$

For convenience, the above Jacobian is partitioned into partial derivatives of the carrier phase $\phi_{n}$, the carrier frequency offset $\omega_{n}$ and the channel impulse response $\mathbf{h}_{n}$, so the following matrix representation of size $1 \times L+2$ is obtained

$$
\mathbf{C}\left[\mathbf{x}_{n}, \mathbf{b}_{n}\right]=\left[\begin{array}{c}
C_{\phi}\left[\mathbf{x}_{n}, \mathbf{b}_{n}\right] \\
C_{\omega}\left[\mathbf{x}_{n}, \mathbf{b}_{n}\right] \\
\mathbf{C}_{\mathbf{h}}^{T}\left[\mathbf{x}_{n}, \mathbf{b}_{n}\right]
\end{array}\right]^{T}
$$

The derivative of the nonlinearity $c\left[\mathbf{x}_{n}, \mathbf{b}_{n}\right]$ according to the phase $\phi_{n}$ results in a scalar value described by

$$
C_{\phi}\left[\mathbf{x}_{n}, \mathbf{b}_{n}\right]=\frac{d c\left[\mathbf{x}_{n}, \mathbf{b}_{n}\right]}{d \phi_{n}}=\mathbf{h}_{n}^{T} \mathbf{b}_{n} j \exp \left\{j \phi_{n}\right\}
$$

at the same moment, the derivative according to the carrier frequency offset $\omega_{n}$ results as follows

$$
C_{\omega}\left[\mathbf{x}_{n}, \mathbf{b}_{n}\right]=\frac{d c\left[\mathbf{x}_{n}, \mathbf{b}_{n}\right]}{d \omega_{n}}=0
$$

And finally, the partial derivative according to the channel vector $\mathbf{h}_{n}$ returns a following vector of size $1 \times L$

$$
\mathbf{C}_{\mathbf{h}}\left[\mathbf{x}_{n}, \mathbf{b}_{n}\right]=\frac{d c\left[\mathbf{x}_{n}, \mathbf{b}_{n}\right]}{d \mathbf{h}_{n}^{T}}=\mathbf{b}_{n}^{T} \exp \left\{j \phi_{n}\right\}
$$

\section{B. Separating Real and Imaginary Components}

We want to note, that the process equation of the dynamical system consists of two real valued parameters: $\phi_{n}$ and $\omega_{n}$ which are related to the residual carrier frequency offset of the measurement equation and the complex valued channel impulse response $\mathbf{h}_{n}$. All these parameters in the process equation distort the transmitted symbols $\left\{b_{n}\right\}$, which are present in the measurement equation $y_{n}$ by the transformation $c\left[\mathbf{x}_{n}, \mathbf{b}_{n}\right]$. Since both, real and complex valued variables are available at the same process equation of the dynamical system, its estimation conducted on the aforementioned measures $y_{n}$ can lead to the complex valued estimates of both $\phi_{n}$ and $\omega_{n}$ carrier parameters. In this case, the time-variant phase offset becomes $\phi_{n}=\phi_{\Re, n}+j \phi_{\Im, n}$ with possibly non-zero imaginary part, which can strictly result in an unwanted additional exponential amplitude distortion of the measurement equation $y_{n}$, according to the following relation

$$
y_{n}=c\left[\mathbf{x}_{n}, \mathbf{b}_{n}\right]+v_{n}=\mathbf{h}_{n}^{T} \mathbf{b}_{n} e^{j \phi_{\Re, n}} e^{-\phi_{\Im, n}}+v_{n}
$$

where $e^{-\phi_{\Im, n}}$ represents the unwanted additional nonlinear relation between the amplitude of the measurement and the imaginary part of the parameter $\phi_{n}$ from the process equation. Since we want to avoid this situation, we adopt the approach for the carrier estimation based on the EKF presented in paper [9] for the joint channel and carrier estimation.

At first, we rewrite the process equation $\mathbf{x}_{n}$ using its new representation $\mathbf{x}_{n}^{I Q}$, which contains separated real and imaginary parts of the channel impulse response $\mathbf{h}_{n}$ according to the following vector of size $2 L+2 \times 1$ representation

$$
\mathbf{x}_{n}^{I Q}=\left[\begin{array}{c}
\phi_{n} \\
\omega_{n} \\
\mathbf{h}_{n}^{I Q}
\end{array}\right]=\left[\begin{array}{c}
\phi_{n} \\
\omega_{n} \\
\Re\left\{\mathbf{h}_{n}\right\} \\
\Im\left\{\mathbf{h}_{n}\right\}
\end{array}\right]
$$

where $\mathbf{h}_{n}^{I Q}=\left[\Re\left\{\mathbf{h}_{n}^{T}\right\}, \Im\left\{\mathbf{h}_{n}^{T}\right\}\right]^{T}$ is a vector of size $2 L \times 1$. Then, we introduce a new representation of the nonlinearity $c\left[\mathbf{x}_{n}, \mathbf{b}_{n}\right]$, which is now a vector of size $2 \times 1$ described by the relation

$$
\mathbf{c}^{I Q}\left[\mathbf{x}_{n}^{I Q}, \mathbf{b}_{n}^{I Q}\right]=\left[\begin{array}{c}
\Re\left\{c\left[\mathbf{x}_{n}, \mathbf{b}_{n}\right]\right\} \\
\Im\left\{c\left[\mathbf{x}_{n}, \mathbf{b}_{n}\right]\right\}
\end{array}\right]
$$


This enabled us to establish a new vector representation of the measurement equation of size $2 \times 1$

$$
\mathbf{y}_{n}^{I Q}=\mathbf{c}^{I Q}\left[\mathbf{x}_{n}^{I Q}, \mathbf{b}_{n}^{I Q}\right]+\mathbf{v}_{n}^{I Q}
$$

where $\mathbf{y}_{n}^{I Q}$ is related with $y_{n}$ according to $\mathbf{y}_{n}^{I Q}=\left[\begin{array}{c}\Re\left\{y_{n}\right\} \\ \Im\left\{y_{n}\right\}\end{array}\right]$ and an additive measurement noise $\mathbf{v}_{n}^{I Q}$, which is related to the measurement noise $v_{n}$ according to $\mathbf{v}_{n}^{I Q}=\left[\begin{array}{c}\Re\left\{v_{n}\right\} \\ \Im\left\{v_{n}\right\}\end{array}\right]$.

For the new representation of the state and measurement components, we can introduce now a new Jacobian matrix $\mathbf{C}^{I Q}\left[\mathbf{x}_{n, r e f}^{I Q}, \mathbf{b}_{n}^{I Q}\right]$ of size $2 \times 2 L+2$ by recalculating partial derivatives of the nonlinearity $\mathbf{c}^{I Q}\left[\mathbf{x}_{n}^{I Q}, \mathbf{b}_{n}^{I Q}\right]$ at the reference point $\mathbf{x}_{n, \text { ref }}^{I Q}$ according to the following relation

$$
\mathbf{C}^{I Q}\left[\mathbf{x}_{n, r e f}^{I Q}, \mathbf{b}_{n}^{I Q}\right]=\left.\frac{d \mathbf{c}^{I Q}\left[\mathbf{x}_{n}^{I Q}, \mathbf{b}_{n}^{I Q}\right]}{\left(d \mathbf{x}_{n}^{I Q}\right)^{T}}\right|_{\mathbf{x}_{n}^{I Q}=\mathbf{x}_{n, r e f}^{I Q}}
$$

As before, we are partitioning the calculation of the derivatives in order to obtain partial results $\mathbf{C}_{\phi}^{I Q}\left[\mathbf{x}_{n}^{I Q}, \mathbf{b}_{n}^{I Q}\right]$ for the phase $\phi_{n}, \mathbf{C}_{\omega}^{I Q}\left[\mathbf{x}_{n}^{I Q}, \mathbf{b}_{n}^{I Q}\right]$ for the carrier frequency offset $\omega_{n}$ and $\mathbf{C}_{\mathbf{h}}^{I Q}\left[\mathbf{x}_{n}^{I Q}, \mathbf{b}_{n}^{I Q}\right]$ for the channel impulse response $\mathbf{h}_{n}^{I Q}$ and we are relating the results with its complex counterparts obtained in the previous section.

First, we calculate the derivative of $\mathbf{c}^{I Q}\left[\mathbf{x}_{n}^{I Q}, \mathbf{b}_{n}^{I Q}\right]$ according to the phase $\phi_{n}$ which results as a vector of size $2 \times 1$ represented by

$$
\mathbf{C}_{\phi}^{I Q}\left[\mathbf{x}_{n}^{I Q}, \mathbf{b}_{n}^{I Q}\right]=\frac{d \mathbf{c}^{I Q}\left[\mathbf{x}_{n}^{I Q}, \mathbf{b}_{n}^{I Q}\right]}{d \phi_{n}}=\left[\begin{array}{c}
\frac{d \mathbf{c}_{\Re, \phi}\left[\mathbf{x}_{n}, \mathbf{b}_{n}\right]}{d \phi_{n}} \\
\frac{d \mathbf{c}_{\Im, \phi}\left[\mathbf{x}_{n}, \mathbf{b}_{n}\right]}{d \phi_{n}}
\end{array}\right]
$$

It can be further calculated for the real and imaginary components of the above partial derivative consecutively as

$$
\begin{aligned}
\frac{d \mathbf{c}_{\Re, \phi}\left[\mathbf{x}_{n}, \mathbf{b}_{n}\right]}{d \phi_{n}}= & -\sin \left(\phi_{n}\right)\left(\mathbf{h}_{\Re, n}^{T} \mathbf{b}_{\Re, n}-\mathbf{h}_{\Im, n}^{T} \mathbf{b}_{\Im, n}\right) \\
& -\cos \left(\phi_{n}\right)\left(\mathbf{h}_{\Re, n}^{T} \mathbf{b}_{\Im, n}+\mathbf{h}_{\Im, n}^{T} \mathbf{b}_{\Re, n}\right) \\
= & \Re\left\{C_{\phi}\left[\mathbf{x}_{n}, \mathbf{b}_{n}\right]\right\}
\end{aligned}
$$

and

$$
\begin{aligned}
\frac{d \mathbf{c}_{\Im, \phi}\left[\mathbf{x}_{n}, \mathbf{b}_{n}\right]}{d \phi_{n}}= & \cos \left(\phi_{n}\right)\left(\mathbf{h}_{\Re, n}^{T} \mathbf{b}_{\Re, n}-\mathbf{h}_{\Im, n}^{T} \mathbf{b}_{\Im, n}\right) \\
& -\sin \left(\phi_{n}\right)\left(\mathbf{h}_{\Re, n}^{T} \mathbf{b}_{\Im, n}+\mathbf{h}_{\Im, n}^{T} \mathbf{b}_{\Re, n}\right) \\
= & \Im\left\{C_{\phi}\left[\mathbf{x}_{n}, \mathbf{b}_{n}\right]\right\}
\end{aligned}
$$

Thus, the derivative $\mathbf{C}_{\phi}^{I Q}\left[\mathbf{x}_{n}^{I Q}, \mathbf{b}_{n}^{I Q}\right]=\frac{d \mathbf{c}^{I Q}\left[\mathbf{x}_{n}^{I Q}, \mathbf{b}_{n}^{I Q}\right]}{d \phi_{n}}$ is related with the derivative $C_{\phi}\left[\mathbf{x}_{n}, \mathbf{b}_{n}\right]=\frac{d \mathbf{c}\left[\mathbf{x}_{n}, \mathbf{b}_{n}\right]}{d \phi_{n}}$ in the following manner

$$
\mathbf{C}_{\phi}^{I Q}\left[\mathbf{x}_{n}^{I Q}, \mathbf{b}_{n}^{I Q}\right]=\left[\begin{array}{c}
\Re\left\{C_{\phi}\left[\mathbf{x}_{n}, \mathbf{b}_{n}\right]\right\} \\
\Im\left\{C_{\phi}\left[\mathbf{x}_{n}, \mathbf{b}_{n}\right]\right\}
\end{array}\right]
$$

To obtain the derivative of $\mathbf{c}^{I Q}\left[\mathbf{x}_{n}^{I Q}, \mathbf{b}_{n}^{I Q}\right]$ according to the carrier frequency offset $\omega_{n}$, represented by the vector $\mathbf{C}_{\omega}^{I Q}\left[\mathbf{x}_{n}^{I Q}, \mathbf{b}_{n}^{I Q}\right]$ of size $2 \times 1$, we introduce the following relation

$$
\begin{aligned}
\mathbf{C}_{\omega}^{I Q}\left[\mathbf{x}_{n}^{I Q}, \mathbf{b}_{n}^{I Q}\right] & =\frac{d \mathbf{c}^{I Q}\left[\mathbf{x}_{n}^{I Q}, \mathbf{b}_{n}^{I Q}\right]}{d \omega_{n}}=\mathbf{0}_{2 \times 1} \\
& =\left[\begin{array}{c}
\Re\left\{C_{\omega}\left[\mathbf{x}_{n}, \mathbf{b}_{n}\right]\right\} \\
\Im\left\{C_{\omega}\left[\mathbf{x}_{n}, \mathbf{b}_{n}\right]\right\}
\end{array}\right]
\end{aligned}
$$

Finally, in order to calculate the partial derivative of the nonlinearity $\mathbf{c}^{I Q}\left[\mathbf{b}_{n}^{I Q}, \mathbf{b}_{n}^{I Q}\right]$ according to the new channel impulse response vector $\mathbf{h}_{n}^{I Q}$ we introduce and then partition the matrix $\mathbf{C}_{\mathbf{h}}^{I Q}\left[\mathbf{x}_{n}^{I Q}, \mathbf{b}_{n}^{I Q}\right]$ of size $2 \times 2 L$ according to

$$
\begin{aligned}
\mathbf{C}_{\mathbf{h}}^{I Q}\left[\mathbf{x}_{n}^{I Q}, \mathbf{b}_{n}^{I Q}\right] & =\frac{d \mathbf{c}^{I Q}\left[\mathbf{x}_{n}^{I Q}, \mathbf{b}_{n}^{I Q}\right]}{d\left(\mathbf{h}_{n}^{I Q}\right)^{T}} \\
& =\left[\begin{array}{ll}
\frac{d c_{\Re, \mathbf{h}}\left[\mathbf{x}_{n}, \mathbf{b}_{n}\right]}{d \mathbf{h}_{\Re, n}^{T}} & \frac{d c_{\Re, \mathbf{h}}\left[\mathbf{x}_{n}, \mathbf{b}_{n}\right]}{d \mathbf{h}_{\Im, n}^{T}} \\
\frac{d c_{\Im, \mathbf{h}}\left[\mathbf{x}_{n}, \mathbf{b}_{n}\right]}{d \mathbf{h}_{\Re, n}^{T}} & \frac{d c_{\Im, \mathbf{h}}\left[\mathbf{x}_{n}, \mathbf{b}_{n}\right]}{d \mathbf{h}_{\Im, n}^{T}}
\end{array}\right]
\end{aligned}
$$

Each component from the partitioned matrix $\mathbf{C}_{\mathbf{h}}^{I Q}\left[\mathbf{x}_{n}^{I Q}, \mathbf{b}_{n}^{I Q}\right]$ is calculated as follows

$$
\begin{aligned}
\frac{d c_{\Re, \mathbf{h}}\left[\mathbf{x}_{n}, \mathbf{b}_{n}\right]}{d \mathbf{h}_{\Re, n}^{T}} & =\mathbf{b}_{\Re, n}^{T} \cos \phi_{n}-\mathbf{b}_{\Im, n}^{T} \sin \phi_{n} \\
& =\Re\left\{\mathbf{C}_{\mathbf{h}}\left[\mathbf{x}_{n}, \mathbf{b}_{n}\right]\right\} \\
\frac{d c_{\Re, \mathbf{h}}\left[\mathbf{x}_{n}, \mathbf{b}_{n}\right]}{d \mathbf{h}_{\Im, n}^{T}} & =-\mathbf{b}_{\Im, n}^{T} \cos \phi_{n}-\mathbf{b}_{\Re, n}^{T} \sin \phi_{n} \\
& =-\Im\left\{\mathbf{C}_{\mathbf{h}}\left[\mathbf{x}_{n}, \mathbf{b}_{n}\right]\right\} \\
\frac{d c_{\Im, \mathbf{h}}\left[\mathbf{x}_{n}, \mathbf{b}_{n}\right]}{d \mathbf{h}_{\Re, n}^{T}} & =\mathbf{b}_{\Im, n}^{T} \cos \phi_{n}+\mathbf{b}_{\Re, n}^{T} \sin \phi_{n} \\
& =\Im\left\{\mathbf{C}_{\mathbf{h}}\left[\mathbf{x}_{n}, \mathbf{b}_{n}\right]\right\} \\
\frac{d c_{\Im, \mathbf{h}}\left[\mathbf{x}_{n}, \mathbf{b}_{n}\right]}{d \mathbf{h}_{\Im, n}^{T}} & =\mathbf{b}_{\Re, n}^{T} \cos \phi_{n}-\mathbf{b}_{\Im, n}^{T} \sin \phi_{n} \\
& \Re\left\{\mathbf{C}_{\mathbf{h}}\left[\mathbf{x}_{n}, \mathbf{b}_{n}\right]\right\}
\end{aligned}
$$

Applying the above results (24)-(27) into (23), we can restate the matrix $\mathbf{C}_{\mathbf{h}}^{I Q}\left[\mathbf{x}_{n}^{I Q}, \mathbf{b}_{n}^{I Q}\right]$ using its complex counterparts from the previous section $\mathbf{C}_{\mathbf{h}}\left[\mathbf{x}_{n}, \mathbf{b}_{n}\right]$ as

$$
\mathbf{C}_{\mathbf{h}}^{I Q}\left[\mathbf{x}_{n}^{I Q}, \mathbf{b}_{n}^{I Q}\right]=\left[\begin{array}{cc}
\Re\left\{\mathbf{C}_{\mathbf{h}}\left[\mathbf{x}_{n}, \mathbf{b}_{n}\right]\right\} & -\Im\left\{\mathbf{C}_{\mathbf{h}}\left[\mathbf{x}_{n}, \mathbf{b}_{n}\right]\right\} \\
\Im\left\{\mathbf{C}_{\mathbf{h}}\left[\mathbf{x}_{n}, \mathbf{b}_{n}\right]\right\} & \Re\left\{\mathbf{C}_{\mathbf{h}}\left[\mathbf{x}_{n}, \mathbf{b}_{n}\right]\right\}
\end{array}\right]
$$

Reassuming, the partial derivative of the nonlinearity which is represented as the matrix of size $2 \times 2 L+2$

$$
\mathbf{C}^{I Q}\left[\mathbf{x}_{n}^{I Q}, \mathbf{b}_{n}^{I Q}\right]=\left[\begin{array}{l}
\mathbf{C}_{\phi}^{I Q, T}\left[\mathbf{x}_{n}^{I Q}, \mathbf{b}_{n}^{I Q}\right] \\
\mathbf{C}_{\omega}^{I Q, T}\left[\mathbf{x}_{n}^{I Q}, \mathbf{b}_{n}^{I Q}\right] \\
\mathbf{C}_{\mathbf{h}}^{I Q, T}\left[\mathbf{x}_{n}^{I Q}, \mathbf{b}_{n}^{I Q}\right]
\end{array}\right]^{T}
$$

can be further restated as follows

$$
\mathbf{C}^{I Q}\left[\mathbf{x}_{n}^{I Q}, \mathbf{b}_{n}^{I Q}\right]=\left[\begin{array}{lll}
\mathbf{C}_{\phi}^{I Q}\left[\mathbf{x}_{n}^{I Q}, \mathbf{b}_{n}^{I Q}\right] & \mathbf{0}_{2 \times 1} & \mathbf{C}_{\mathbf{h}}^{I Q}\left[\mathbf{x}_{n}^{I Q}, \mathbf{b}_{n}^{I Q}\right]
\end{array}\right]
$$

\section{Algorithm DESCRIPTION}

The extended Kalman filter is a well known method in iterative estimation of a state vector in a nonlinear model of a dynamical system [4], [10]. On the contrary to the Kalman filtering, which is designed for the linear models, the EKF can handle nonlinearities thanks to the linearisation procedure [4], [10], thus it provides an approximation to the optimal nonlinear estimation [4], [10]. In this section, the application of the EKF for the joint channel and carrier estimation is provided.

Prediction of the process equation of the dynamics at time instant $n$ is described by the vector of size $2 L+2 \times 1$

$$
\hat{\mathbf{x}}_{n \mid n-1}^{I Q}=\mathbf{A}^{I Q} \hat{\mathbf{x}}_{n-1 \mid n-1}^{I Q}
$$




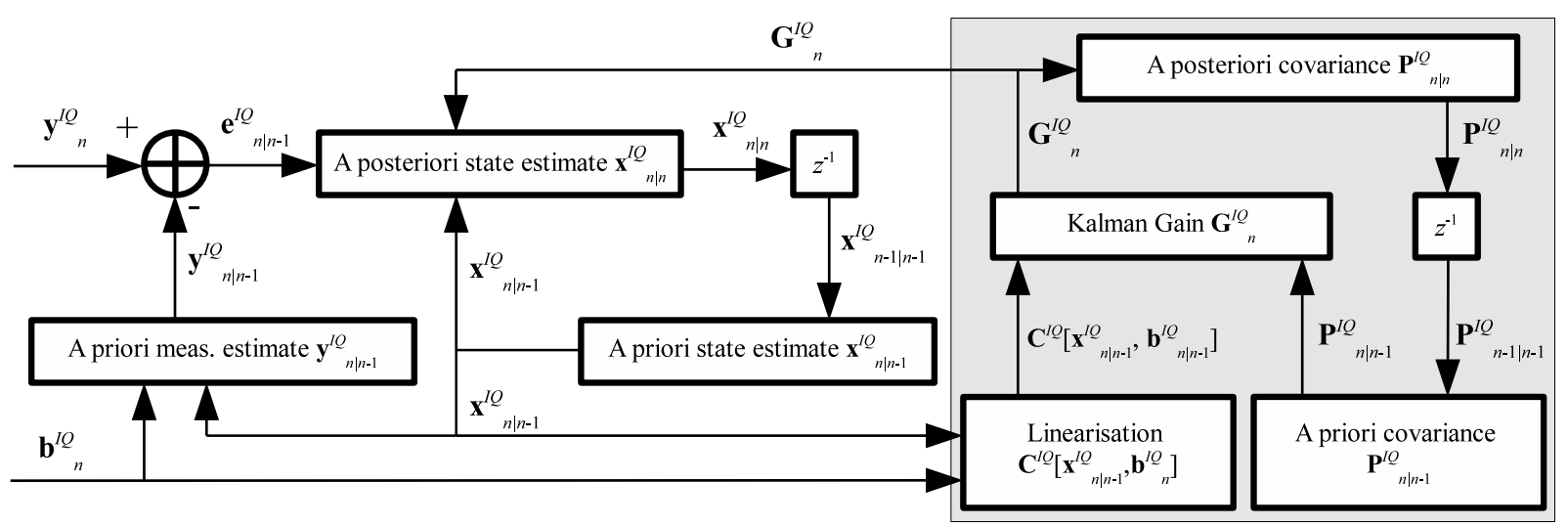

Fig. 1. Block diagram of the channel and carrier estimator based on the EKF

where the transition matrix $\mathbf{A}^{I Q}$ of size $2 L+2 \times 2 L+2$ is related with the transition matrix $\mathbf{A}$ of size $L+2 \times L+2$ by the following relation

$$
\mathbf{A}^{I Q}=\left[\begin{array}{cc}
\mathbf{A} & \mathbf{0}_{L+2 \times L} \\
\mathbf{0}_{L \times L+2} & \mathbf{A}_{\mathbf{h}}
\end{array}\right]
$$

where the channel model is assumed $\mathbf{A}_{\mathbf{h}}=\mathbf{I}_{L \times L}$.

The error of the a priori estimate of the process equation $\mathbf{x}_{n \mid n-1}^{I Q}$ according to the true state $\mathbf{x}_{n}^{I Q}$ is defined by

$$
\varepsilon_{n \mid n-1}^{I Q}=\mathbf{x}_{n}^{I Q}-\mathbf{x}_{n \mid n-1}^{I Q}
$$

In the Extended Kalman Filter it is assumed [4], [10], that the reference point is equal to the a priori estimate of the process equation

$$
\mathbf{x}_{n, r e f}=\mathbf{x}_{n \mid n-1}
$$

From (15), the nonlinear relation $\mathbf{c}^{I Q}\left[\hat{\mathbf{x}}_{n \mid n-1}^{I Q}, \mathbf{b}_{n}^{I Q}\right]$ between the a priori estimate of the process equation and the a priori estimate of the measurement $\mathbf{y}_{n \mid n-1}^{I Q}$ can be defined by the vector of size $2 \times 1$ of the form

$$
\mathbf{c}^{I Q}\left[\hat{\mathbf{x}}_{n \mid n-1}^{I Q}, \mathbf{b}_{n}^{I Q}\right]=\left[\begin{array}{l}
\Re\left\{c\left[\hat{\mathbf{x}}_{n \mid n-1}, \mathbf{b}_{n}\right]\right\} \\
\Im\left\{c\left[\hat{\mathbf{x}}_{n \mid n-1}, \mathbf{b}_{n}\right]\right\}
\end{array}\right]
$$

where $c\left[\hat{\mathbf{x}}_{n \mid n-1}, \mathbf{b}_{n}\right]=\mathbf{h}_{n \mid n-1}^{T} \mathbf{b}_{n} \exp \left\{j \phi_{n \mid n-1}\right\}$. Thus, the a priori measurement equation is defined as follows

$$
\mathbf{y}_{n \mid n-1}^{I Q}=\mathbf{c}^{I Q}\left[\hat{\mathbf{x}}_{n \mid n-1}^{I Q}, \mathbf{b}_{n}^{I Q}\right]
$$

In order to linearise the nonlinear relation between the state and the measurement equation, the following Jacobian matrix followed from (17) and (30) under the assumption (34) is used:

$$
\mathbf{C}^{I Q}\left[\mathbf{x}_{n \mid n-1}^{I Q}, \mathbf{b}_{n}^{I Q}\right]=\left[\begin{array}{c}
\mathbf{C}_{\phi}^{I Q}\left[\mathbf{x}_{n \mid n-1}^{I Q}, \mathbf{b}_{n}^{I Q}\right]^{T} \\
\mathbf{0}_{1 \times 2} \\
\mathbf{C}_{\mathbf{h}}^{I Q}\left[\mathbf{x}_{n \mid n-1}^{I Q}, \mathbf{b}_{n}^{I Q}\right]^{T}
\end{array}\right]^{T}
$$

The error covariance matrix of size $2 L+2 \times 2 L+2$ of the a priori estimator error for the EKF is then

$$
\mathbf{P}_{n \mid n-1}^{I Q}=\mathbf{A}^{I Q} \mathbf{P}_{n-1 \mid n-1}^{I Q}\left(\mathbf{A}^{I Q}\right)^{T}+\mathbf{Q}_{-1}^{I Q}
$$

The Kalman gain at time instant $n$ is described by the matrix of size $2 L+2 \times 2$ of the following form

$$
\begin{aligned}
\mathbf{G}_{n}^{I Q}= & \mathbf{P}_{n \mid n-1}^{I Q} \mathbf{C}^{I Q, T}\left[\hat{\mathbf{x}}_{n \mid n-1}^{I Q}, \mathbf{b}_{n}^{I Q}\right] \times \\
& \left(\mathbf{C}^{I Q}\left[\hat{\mathbf{x}}_{n \mid n-1}^{I Q}, \mathbf{b}_{n}^{I Q}\right] \mathbf{P}_{n \mid n-1}^{I Q} \mathbf{C}_{\phi}^{I Q, T}\left[\hat{\mathbf{x}}_{n \mid n-1}^{I Q}, \mathbf{b}_{n}^{I Q}\right]\right. \\
& \left.+\mathbf{R}_{-1}^{I Q}\right)^{-1}
\end{aligned}
$$

The a priori estimation error of the measurement equation $\mathbf{y}_{n \mid n-1}^{I Q}$ according to the true measure $\mathbf{y}_{n}^{I Q}$ is described by the following difference

$$
\mathbf{e}_{n \mid n-1}^{I Q}=\mathbf{y}_{n}^{I Q}-\mathbf{y}_{n \mid n-1}^{I Q}
$$

Now, the a posteriori estimate of the process equation $\hat{\mathbf{x}}_{n \mid n}^{I Q}$ is the vector of size $2 L+2 \times 1$ of the following form

$$
\hat{\mathbf{x}}_{n \mid n}^{I Q}=\hat{\mathbf{x}}_{n \mid n-1}^{I Q}+\mathbf{G}_{n}^{I Q}\left(\mathbf{y}_{n}^{I Q}-\mathbf{c}^{I Q}\left[\hat{\mathbf{x}}_{n \mid n-1}^{I Q}, \mathbf{b}_{n}^{I Q}\right]\right)
$$

The error covariance matrix of size $2 L+2 \times 2 L+2$ of the a posteriori state estimate at time instant $n$ is described by

$$
\mathbf{P}_{n \mid n}^{I Q}=\left(\mathbf{I}_{2 L+2}-\mathbf{G}_{n}^{I Q} \mathbf{C}^{I Q}\left[\hat{\mathbf{x}}_{n \mid n-1}^{I Q}, \mathbf{b}_{n}^{I Q}\right]\right) \mathbf{P}_{n \mid n-1}^{I Q}
$$

Thus, all the equations for the joint carrier and channel estimation using the EKF are presented above.

1) Initialization: For the initialization purposes, at time instant $n=0$ the state noise covariance matrix $\mathbf{Q}_{-1}^{I Q}$, the measurement noise covariance matrix $\mathbf{R}_{-1}^{I Q}$ and the a posteriori state error covariance matrix $\mathbf{P}_{-1 \mid-1}^{I Q}$ must be provided.

At first, the state noise covariance matrix $\mathbf{Q}_{-1}^{I Q}$ will be defined. We assume, that each element of the state noise process represented by the vector $\mathbf{w}_{n}^{I Q}$ of size $2 L+2 \times 1$ is statistically independent random process modelled by the AWGN [3], [9], [10]. Under the above assumption, the state noise covariance matrix is a diagonal matrix of size $2 L+2 \times 2 L+2$ defined as

$$
\begin{aligned}
\mathbf{Q}_{-1}^{I Q} & =E\left\{\mathbf{w}_{n}^{I Q} \mathbf{w}_{n}^{I Q, T}\right\} \\
& =\operatorname{diag}\left\{\sigma_{w_{\phi}}^{2}, \sigma_{w_{\omega}}^{2}, \boldsymbol{\sigma}_{\mathbf{h}^{I Q}}^{2}\right\}
\end{aligned}
$$

where $\sigma_{w_{\phi}}^{2}$ and $\sigma_{w_{\omega}}^{2}$ is an initial phase and frequency variance and $\sigma_{\mathbf{h}^{I Q}}^{2}$ is an initial variance of the channel taps defined by 
the vector of size $2 L \times 1$ of the following form

$$
\begin{aligned}
\boldsymbol{\sigma}_{\mathbf{h}^{I Q}}^{2}= & {\left[\sigma_{w_{h_{\Re}(0)}}^{2}, \ldots, \sigma_{w_{h_{\Re}(L-1)}}^{2},\right.} \\
& \left.\sigma_{w_{h_{\Im}(0)}}^{2}, \ldots, \sigma_{w_{h_{\Im}(L-1)}}^{2}\right]^{T}
\end{aligned}
$$

Under the assumption, that the measurement noise vector $\mathbf{v}_{n}^{I Q}$ is AWGN, the measurement noise covariance matrix is defined by a diagonal matrix of size $2 \times 2$ as

$$
\mathbf{R}_{-1}^{I Q}=E\left\{\mathbf{v}_{n}^{I Q} \mathbf{v}_{n}^{I Q, T}\right\}=\operatorname{diag}\left\{\sigma_{v}^{2}, \sigma_{v}^{2}\right\}
$$

Following the assumptions presented in [3] and [8] for the channel estimation and the synchronization purposes using the Kalman filtering, we propose to assume the a posteriori state error covariance matrix in the initialization stage to be the diagonal matrix of size $2 L+2 \times 2 L+2$ described as

$$
\begin{aligned}
\mathbf{P}_{-1 \mid-1}^{I Q} & =E\left\{\varepsilon_{-1 \mid-1}^{I Q} \varepsilon_{-1 \mid-1}^{I Q, T}\right\} \\
& =\operatorname{diag}\left\{\sigma_{\varepsilon_{\phi},-1 \mid-1}^{2}, \sigma_{\varepsilon_{\omega},-1 \mid-1}^{2}, \boldsymbol{\sigma}_{\epsilon_{\mathbf{h}_{-1 \mid-1}}^{2}}^{2}\right\}
\end{aligned}
$$

where the channel estimation covariance error $\boldsymbol{\sigma}_{\epsilon_{\mathbf{h}_{-1 \mid-1}}^{2}}^{2}$ is defined by the following vector of size $2 L \times 1$

$$
\begin{aligned}
\boldsymbol{\sigma}_{\epsilon_{\mathbf{h}_{-1 \mid-1}}}^{2}= & {\left[\sigma_{\varepsilon_{\mathbf{h}_{\Re,-1 \mid-1}}}^{2}(0), \ldots, \sigma_{\varepsilon_{\mathbf{h}_{\Re,-1 \mid-1}}^{2}}^{2}(L-1),\right.} \\
& \left.\sigma_{\varepsilon_{\mathbf{h}_{\Im,-1 \mid-1}}^{2}}^{2}(0), \ldots, \sigma_{\varepsilon_{\mathbf{h}_{\Im,-1 \mid-1}}}^{2}(L-1)\right]^{T}
\end{aligned}
$$

\section{Algorithm Summary}

Proposed algorithm can be presented by the block diagram depicted in Fig. 1. In order to summarize its main stages, it can be divided into the initialization and the Kalman recursion in the following way

\section{- Initialization:}

- A posteriori estimate of the process equation $(2 L+$ $2 \times 1)$

$$
\mathbf{x}_{-1 \mid-1}^{I Q}=\mathbf{0}_{2 L+2 \times 1}
$$

- A posteriori state error covariance $(2 L+2 \times 2 L+2)$

$$
\mathbf{P}_{-1 \mid-1}^{I Q}=\operatorname{diag}\left\{\sigma_{\epsilon_{\phi_{-1 \mid-1}}}, \sigma_{\epsilon_{\omega_{-1 \mid-1}}}, \sigma_{\epsilon_{-1 \mid-1}^{I Q}}^{2}\right\}
$$

- Measurement noise covariance $(2 \times 2)$

$$
\mathbf{R}_{-1}^{I Q}=\operatorname{diag}\left\{\sigma_{v}^{2}, \sigma_{v}^{2}\right\}
$$

- State noise covariance $(2 L+2 \times 2 L+2)$

$$
\mathbf{Q}_{-1}^{I Q}=\operatorname{diag}\left\{\sigma_{w_{\phi}}^{2}, \sigma_{w_{\omega}}^{2}, \sigma_{w_{\mathbf{h}} I Q}^{2}\right\}
$$

- Kalman recursion for $n=0,1,2, \ldots$.

- Time update step:

$$
\begin{gathered}
\mathbf{x}_{n \mid n-1}^{I Q}=\mathbf{A}^{I Q} \mathbf{x}_{n-1 \mid n-1}^{I Q} \\
\mathbf{P}_{n \mid n-1}^{I Q}=\mathbf{A}^{I Q} \mathbf{P}_{n-1 \mid n-1}^{I Q} \mathbf{A}^{I Q, T}+\mathbf{Q}_{-1}^{I Q}
\end{gathered}
$$

- Kalman gain computation:

$$
\begin{aligned}
\mathbf{G}_{n}^{I Q}= & \mathbf{P}_{n \mid n-1}^{I Q} \mathbf{C}_{n \mid n-1}^{I Q} \times \\
& \left(\mathbf{C}_{n \mid n-1}^{I Q} \mathbf{P}_{n \mid n-1}^{I Q} \mathbf{C}_{n \mid n-1}^{I Q, T}+\mathbf{R}_{-1}^{I Q}\right)^{-1}
\end{aligned}
$$

- Measurement update step:

$$
\begin{gathered}
\mathbf{x}_{n \mid n}^{I Q}=\mathbf{x}_{n \mid n-1}^{I Q}+\mathbf{G}_{n}^{I Q}\left(\mathbf{y}_{n}^{I Q}-\mathbf{y}_{n \mid n-1}^{I Q}\right) \\
\mathbf{P}_{n \mid n}^{I Q}=\left(\mathbf{I}-\mathbf{G}_{n}^{I Q} \mathbf{C}_{n \mid n-1}^{I Q}\right) \mathbf{P}_{n \mid n-1}^{I Q}
\end{gathered}
$$

\section{- Nonlinearity and linearization of the state-space rep- resentation:}

- Nonlinearity between the a posteriori estimate of the state and measure: $\mathbf{y}_{n \mid n-1}^{I Q}=\mathbf{c}^{I Q}\left[\mathbf{x}_{n \mid n-1}^{I Q}, \mathbf{b}_{n}^{I Q}\right]$

- Jacobian matrix: $\mathbf{C}_{n \mid n-1}^{I Q}=\mathbf{C}^{I Q}\left[\mathbf{x}_{n \mid n-1}^{I Q}, \mathbf{b}_{n}^{I Q}\right]$

\section{Simulation Results}

The proposed algorithm for joint channel and carrier estimation, further referenced as the Joint Extended Kalman Filter (JEKF) is compared with the separate Kalman channel estimator [3] concatenated with the extended Kalman carrier recovery [9], which is referenced as the Separate Extended Kalman Filter (SEKF).

The communication channel of length $L$ is assumed to be a single frame time invariant, generated from an uncorrelated and zero mean stationary random process with Gaussian distribution. The channel impulse response has normalized energy, which is $\sum_{l=0}^{L-1}\left|h_{l}\right|^{2}=1$ Additionally, the AWGN is present in the received signal with signal to noise ratio $S N R=80 \mathrm{~dB}$, except the simulation, in which a relation between the MSE and the SNR is estimated.

In order to calculate the mean square error of the a posteriori estimate of the measurement equation is defined as

$$
y_{n \mid n}=\mathbf{h}_{n \mid n}^{T} \mathbf{b}_{n} \exp \left\{j \phi_{n \mid n}\right\}
$$

Estimation of the MSE is performed using the Monte Carlo (MC) simulations based on $M=1000$ runs, and the estimate is calculated as

$$
\operatorname{MSE}(n)=\frac{1}{M} \sum_{m=0}^{M-1}\left|e_{n \mid n}^{(m)}\right|^{2}=\frac{1}{M} \sum_{m=0}^{M-1}\left|y_{n}-y_{n \mid n}^{(m)}\right|^{2}
$$

where the upper index $(m)$ represents the number of the $\mathrm{MC}$ run.

At first, simulations were focused on the initialization behaviour of the algorithms for different values of the initialization parameters $\mathbf{R}_{-1}^{I Q}, \mathbf{Q}_{-1}^{I Q}, \mathbf{P}_{-1 \mid-1}^{I Q}$ and its influence to the speed of adaptation and the steady state mean square error of the a posteriori estimate of the measurement $y_{n \mid n}$ for both the JEKF and the SEKF with the same initialization parameters. The channel length is assumed to be $L=8$ and the residual carrier frequency offset $f_{0}=\omega_{0} / 2 \pi$ normalized to the symbol frequency $f_{s}$ is equal to $f_{0} / f_{s}=0.01$.

In Figure 2(a) an influence of the state noise covariance matrix parameter is investigated for initial values $\mathbf{Q}_{-1}^{I Q}=\left\{10^{-2} \mathbf{I}, 10^{-3} \mathbf{I}, 10^{-4} \mathbf{I}\right\}$ with the fixed measurement noise covariance $\mathbf{R}_{-1}^{I Q}=0.25 \mathbf{I}$ and the a posteriori state error covariance $\mathbf{P}_{-1 \mid-1}^{I Q}=10^{-3} \mathbf{I}$. When the state noise covariance matrix is $\mathbf{Q}_{-1}^{I Q}=10^{-2} \mathbf{I}$, the JEKF reaches better results compared with the SEKF in both the speed of 


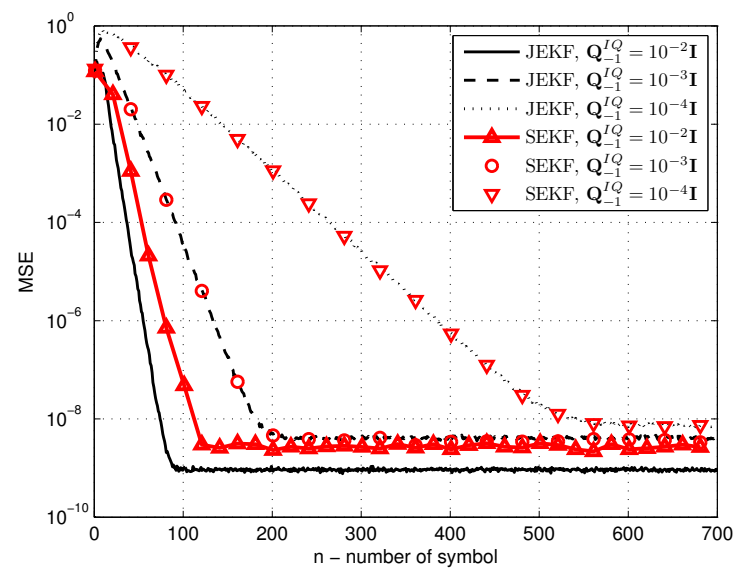

(a) Initial parameters $\mathbf{P}_{-1 \mid-1}^{I Q}=10^{-3} \mathbf{I}, \mathbf{R}_{-1}^{I Q}=0.25 \mathbf{I}$

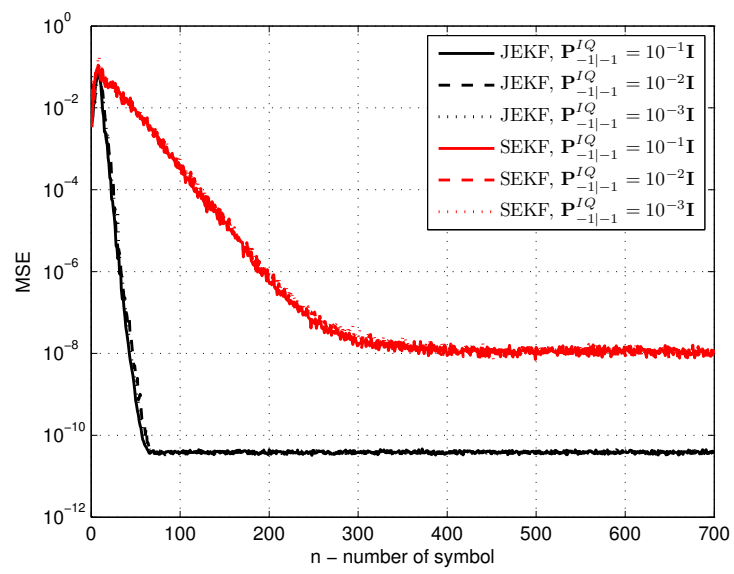

(b) Initial parameters $\mathbf{Q}_{-1}^{I Q}=10^{-2} \mathbf{I}, \mathbf{R}_{-1}^{I Q}=0.025 \mathbf{I}$

Fig. 2. Estimated mean square error of $\mathbf{y}_{n \mid n}^{I Q}$ for random channel of length $L=8$ with the normalized carrier frequency offset $f_{0} / f_{s}=0.01$

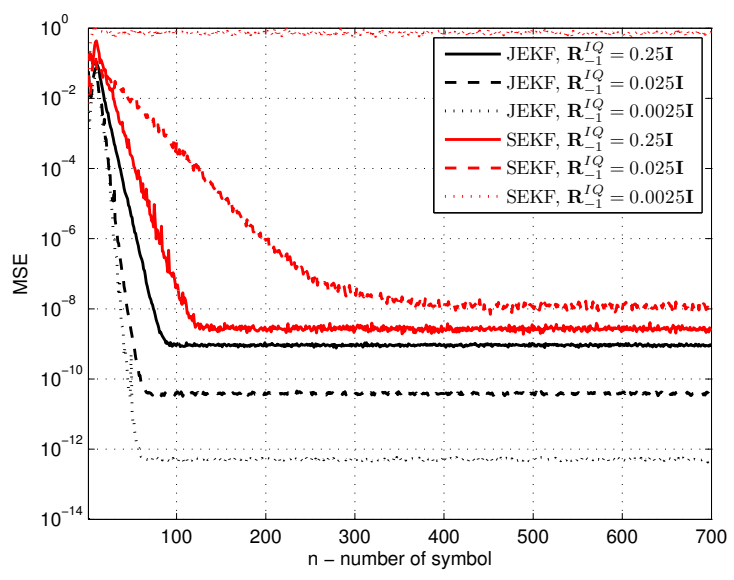

(a) Initial parameters $\mathbf{Q}_{-1}^{I Q}=10^{-2} \mathbf{I}, \mathbf{P}_{-1 \mid-1}^{I Q}=10^{-3} \mathbf{I}$, normalized carrier frequency offset $f_{0} / f_{s}=0.01$ and random channel of length $L=8$

Fig. 3. Estimated mean square error of $\mathbf{y}_{n \mid n}^{I Q}$

adaptation and the steady state MSE. However, when the measurement noise covariance matrix is $\mathbf{Q}_{-1}^{I Q}=\left\{10^{-3} \mathbf{I}, 10^{-4} \mathbf{I}\right\}$, both algorithms behaves in a similar way.

In Figure 2(b) an influence of the measurement error covariance matrix $\mathbf{P}_{-1 \mid-1}^{I Q}$ is checked for the speed of adaptation and the steady state MSE is investigated. For simulation purposes, the measurement noise covariance matrix is assumed $\mathbf{R}_{-1}^{I Q}=0.025 \mathbf{I}$ and the state noise covariance matrix is $\mathbf{Q}_{-1}^{I Q}=10^{-2} \mathbf{I}$. According to [3], this parameter in the Kalman channel estimator determines the speed of adaptation, however, for the assumed range of the parameter $\mathbf{P}_{-1 \mid-1}^{I Q}=\left\{10^{-1} \mathbf{I}, 10^{-2} \mathbf{I}, 10^{-3} \mathbf{I}\right\}$ the speed of adaptation does not change significantly and the steady state MSE estimates remains at the similar level.

In Figure 3(a) an influence of the measurement noise covariance matrix $\mathbf{R}_{-1}$ is investigated consecutively for $\mathbf{Q}_{-1}^{I Q}=10^{-2} \mathbf{I}, \mathbf{P}_{-1 \mid-1}^{I Q}=10^{-2} \mathbf{I}$ and $\mathbf{Q}_{-1}^{I Q}=10^{-2}$,

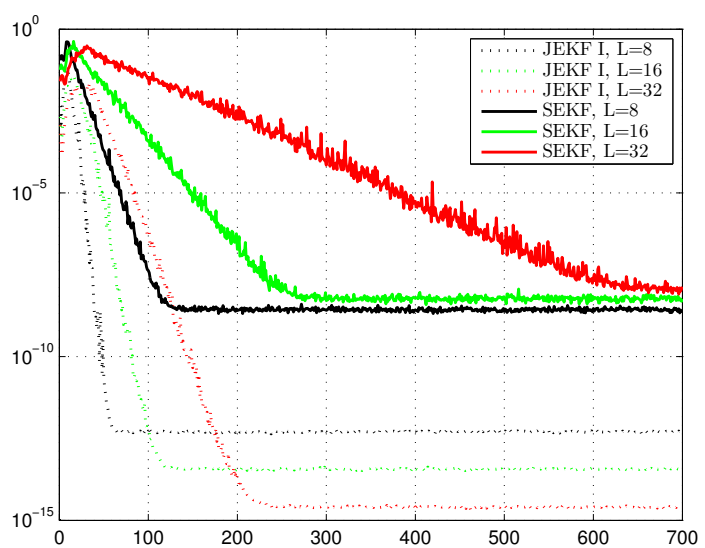

(b) Normalized carrier frequency offset $f_{0} / f_{s}=0.025$ and random channel of length $L=\{8,16,32\}$

$\mathbf{P}_{-1 \mid-1}^{I Q}=10^{-3} \mathbf{I}$. For the simulation purposes, the measurement noise covariance $\mathbf{R}_{-1 \mid-1}=\{0.25 \mathbf{I}, 0.025 \mathbf{I}, 0.0025 \mathbf{I}\}$. For the JEKF, decreasing the initial value of the measurement noise covariance improved both the speed of adaptation and the steady state mean square error. However, for the SEKF and the same initial parameters, the speed of adaptation and the steady state mean square error is increasing for decreasing the initial value of measurement noise covariance matrix $\mathbf{R}_{-1}^{I Q}$.

For further experiments, the initialization parameters are chosen from the results depicted in Figs. 2(a), 2(b) and 3(a) for the best performance of the algorithms. Both of the JEKF configurations have the same a posteriori estimation error covariance matrix $\mathbf{P}_{-1 \mid-1}^{I Q}=10^{-3} \mathbf{I}$ and measurement noise covariance matrix $\mathbf{R}_{-1}^{I Q}=0.0025 \mathbf{I}$, but the state noise covariance for the JEKF I is $\mathbf{Q}_{-1}^{I Q}=10^{-2} \mathbf{I}$, and for the JEKF II is $\mathbf{Q}_{-1}^{I Q}=10^{-3} \mathbf{I}$. In case of the SEKF, the state 


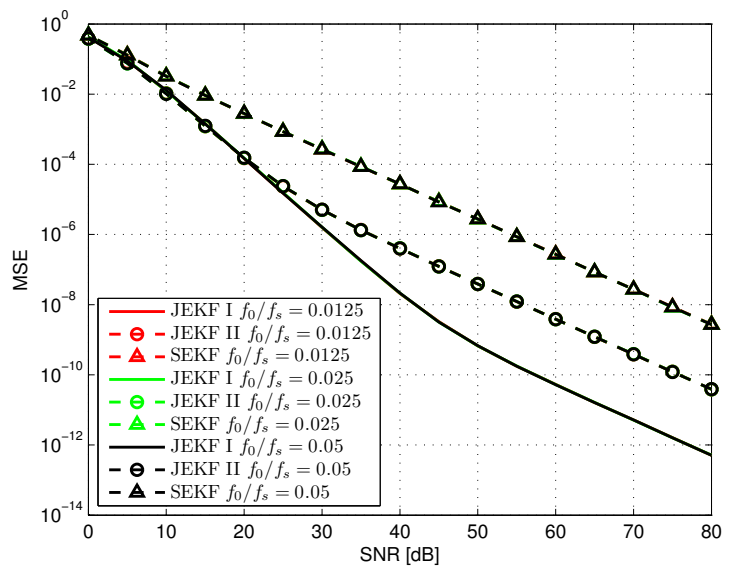

(a) Estimated MSE $=f(S N R)$ for normalized carrier frequency offset $f_{0} / f_{s}=\{0.0125,0.025,0.05\}$ and random channel of length $L=8$

Fig. 4. Estimated mean square error of $\mathbf{y}_{n \mid n}^{I Q}$

noise covariance is $\mathbf{Q}_{-1}^{I Q}=10^{-3} \mathbf{I}$, the a posteriori estimation error covariance $\mathbf{P}_{-1 \mid-1}^{I Q}=10^{-3} \mathbf{I}$ and the measurement noise covariance $\mathbf{R}_{-1}^{I Q}=0.025 \mathbf{I}$.

At first, an influence of different values of the channel length assumed to be $L=\{8,16,32\}$ at the same normalized carrier frequency offset $f_{0} / f_{s}=0.025$ for a speed of adaptation is investigated. From the simulation results depicted in Fig. 3(b) it can be seen, that when both the channel and the vector $\mathbf{x}_{n}^{I Q}$ length is increasing, the speed of adaptation degrades for both the JEKF I and the SEKF. However, the JEKF I performs better than the SEKF for the same length. Additionally, it can be noted, that the steady state MSE for the JEKF I is lowering for higher length of the process vector $\mathbf{x}_{n}^{I Q}$ and the channel impulse response. On the other hand, the steady state MSE of the SEKF is degrading when the channel length and the dimension of the vector $\mathbf{x}_{n}^{I Q}$ is increasing. The steady state $M S E=f(S N R)$ is investigated with more details at the end of this chapter.

Next, the estimated MSE in the steady state as a function of SNR is presented for the JEKF I, JEKF II and the SEKF. The results of simulations are depicted in Fig. 4(a) for the channel length $L=8$ and the normalized carrier frequency offset $f_{0} / f_{s}=\{0.0125,0.025,0.05\}$. It can be seen, that the JEKF I and the JEKF II perform better than the SEKF and the best results of the MSE are obtained for the JEKF I. However, the advantage of two JEKF configurations over the SEKF is gradually decreasing, when the value of the SNR is getting smaller.

At the end, we investigated an influence of the channel length to the estimated MSE in the steady state as a function of the signal to noise ratio (SNR) for the JEKF I and the SEKF. The channel length is assumed to be $L=\{8,16,32\}$ and the normalized carrier frequency offset $f_{0} / f_{s}=0.025$. It can be seen from the simulation results depicted in Fig. 4(b), that in case of the JEKF I, the steady state MSE is decreasing for higher length of the impulse response, i.e. for $L=8$ at $S N R=23 \mathrm{~dB}$ the MSE is approximately at the same level as

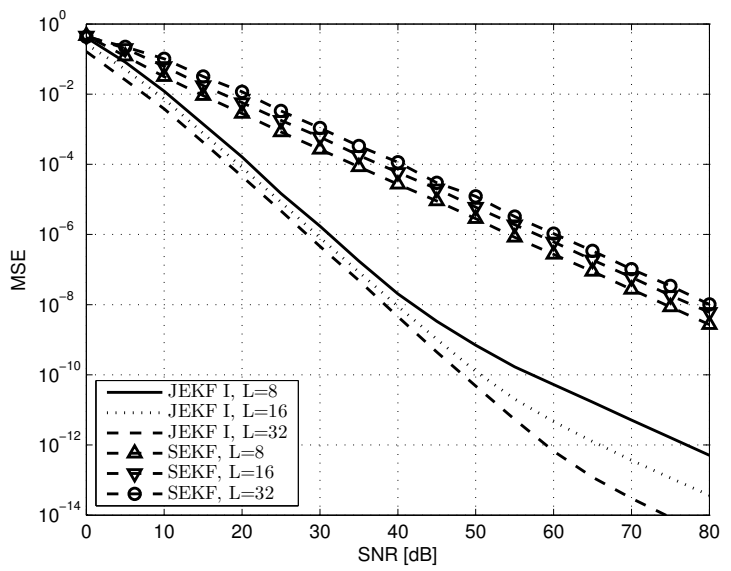

(b) Estimated MSE $=f(S N R)$ for normalized carrier frequency offset $f_{0} / f_{s}=0.025$ and random channel of length $L=\{8,16,32\}$

for the channel length $L=16$ at $S N R=21.5 \mathrm{~dB}$ and for the channel length $L=32$ at $S N R=20 \mathrm{~dB}$. In case of the SEKF, when the channel length increases from $L=8$ to $L=16$ and then, to $L=32$, the characteristics of the $M S E=f(S N R)$ degrades for about $2.5 \mathrm{~dB}$ at each step.

\section{CONCLUSION}

In this paper the joint channel and carrier estimation technique based on the EKF is proposed. The algorithm is compared with concatenated the KF channel [3] and the EKF carrier estimator [9] in which the state-space models are modified in order to incorporate both types of distortions. For both algorithms the Monte Carlo simulations were performed in order to compare the speed of adaptation and the steady state MSE. The joint channel and carrier estimation using the extended Kalman filter can provide better results than the SEKF and it seems to be an interesting topic for further studies.

\section{REFERENCES}

[1] H. Meyr, M. Moeneclaey, and S. A. Fechtel, Digital Communication Receivers. John Willey \& Sons, 1998.

[2] K. Wesołowski, Podstawy cyfrowych systemow telekomunikacyjnych. Warszawa: WKL, 2003.

[3] L. Hanzo, C. H. Wong, and M. S. Yee, Adaptive wireless transceivers. John Willey \& Sons, 2002.

[4] S. Haykin, Adaptive Filter Theory. Prentice Hall, 1997.

[5] S. Benedetto and E. Biglieri, Principles of Digital Transmission With Wireless Applications. New York: Kluwer Academic/Plenum Publishers, 1999.

[6] J. G. Proakis, Digital Communications. New York: Mc Graw-Hill, 1996.

[7] B. Chun and Y. H. Lee, "Design of Variable Loop Gains of DualLoop DPLL," IEEE Transactions on Communications, vol. 45, no. 12, December 1997.

[8] P. F. Driessen, "DPLL Bit Synchronizer with Rapid Acquisition Using Adaptive Kalman Filtering Techniques," IEEE Transactions on Communications, vol. 42, no. 9, September 1994.

[9] W. T. Lin and D. C. Chang, "Adaptive Carrier Synchronization Using Decision-Aided Kalman Filtering Algorithms," IEEE Transactions on Consumer Electronics, vol. 53, no. 4, November 2007.

[10] S. Haykin, Kalman Filtering and Neural Networks. Prentice Hall, 1997. 